Cahiers de recherches médiévales

Journal of medieval studies

$8 \mid 2001$

La protection spirituelle au Moyen Âge

\title{
Un monastère et son patron
}

Saint Benoît, patron et protecteur de l'abbaye de Fleury

\section{Anselme Davril}

\section{OpenEdition}

Journals

Édition électronique

URL : https://journals.openedition.org/crm/382

DOI : $10.4000 / \mathrm{crm} .382$

ISSN : 1955-2424

Éditeur

Honoré Champion

Édition imprimée

Date de publication : 15 janvier 2001

Pagination : 43-55

ISSN : 1272-9752

Référence électronique

Anselme Davril, « Un monastère et son patron », Cahiers de recherches médiévales [En ligne], 8| 2001, mis en ligne le 30 novembre 2009, consulté le 15 décembre 2022. URL : http://

journals.openedition.org/crm/382; DOI : https://doi.org/10.4000/crm.382

Ce document a été généré automatiquement le 15 décembre 2022.

Tous droits réservés 


\section{Un monastère et son patron}

Saint Benoît, patron et protecteur de l'abbaye de Fleury

\section{Anselme Davril}

1 De tout temps, et de nos jours encore, tout monastère a son saint patron. Mais actuellement on parlera plutôt de titulaire et ce titulaire pourra au besoin être changé, sans que cela ne pose de problème. Ainsi, une communauté de moniales qui s'est récemment transférée d'un lieu à un autre a trouvé tout à fait normal de changer de patronage à cette occasion.

2 Au Moyen Âge, il n'en était pas ainsi, mais, comme l'a magistralement montré Peter Brown, le saint patron: " avait pris tous les traits d'un patronus de la Rome tardive. Le saint était le bon patronus: c'était le patronus qui intercédait avec succès, dont la richesse était à la disposition de tous, dont la potentia s'exerçait sans violence et à qui chacun pouvait sans y être contraint prouver sa loyauté1. "

3 Et le patronage exercé par le saint est d'autant plus efficace que l'on a pu s'assurer la possession de son corps ou d'une partie au moins de ses reliques, car : «leur corps terrestre, rempli de Dieu de leur vivant, reste, après leur mort, imprégné de substance divine agissante, de virtus, comme en témoignent les nombreux miracles dont leurs tombeaux sont le théatre ${ }^{2}$.» On croyait donc que le saint monté au ciel était présent dans sa sépulture terrestre. C'est cette croyance qui explique les très nombreuses translations de saints qui ont eu lieu au haut Moyen Âge.

4 C'est l'un de ces cas de patronage que l'on voudrait examiner ici, celui du patronage de saint Benoît sur le monastère de Fleury, tel qu'il nous apparaît à travers les Miracula sancti Benedicti qui racontent les miracles, disons plutôt les faits merveilleux, survenus auprès des reliques de saint Benoît conservées à l'abbaye de Fleury et en différents lieux dépendants de Fleury, ou plutôt, comme disent nos auteurs, lieux appartenant à saint Benoît. C'est un recueil de neuf livres écrits par cinq auteurs, tous moines de Fleury, entre le IX ${ }^{\mathrm{e}}$ et le XII ${ }^{\mathrm{e}}$ siècles. Le livre I, dû à Adrevald qui écrivait au milieu du $\mathrm{IX}^{\mathrm{e}}$ siècle, fait suite au récit de la translation des reliques de saint Benoît et est suivi d'un appendice de deux chapitres rédigés par son confrère Adelier. Après un intervalle de près d'un siècle, Aimoin reprit la rédaction des Miracula dans les premières années $\mathrm{du} \mathrm{XI}^{\mathrm{e}}$ siècle et rédigea les livres II et III. Après sa mort, vers 1010, l'œuvre fut reprise 
par son confrère André, futur prieur, qui rédigea les livres IV à VII. Un livre VIII fut ensuite l'œuvre de Raoul dit Le Tourtier (ou Tortaire) qui écrit dans la seconde moitié du XI ${ }^{e}$ siècle et le début du XII ${ }^{e}$ (il est mort en 1122). Enfin le livre IX est l'œuvre d'Hugues de Sainte-Marie qui vivait au cours du XII siècle. Remarquons d'ailleurs que ces neuf livres ne sont pas une simple chronique du pèlerinage qui se contenterait de relater les miracles accomplis auprès des reliques de saint Benoît ou par saint Benoît. Les choses sont beaucoup plus complexes car nos moines sont plus historiens que chroniqueurs et la rédaction des Miracula n'est souvent pour eux que prétexte à grandes fresques historiques ${ }^{3}$.

Que saint Benoît soit le patron et le protecteur de l'abbaye de Fleury, tout le recueil des Miracula est destiné à le proclamer. Mais saint Benoît intervient lui-même pour l'affirmer très explicitement dans une vision dont est gratifié le sacristain de la basilique le jour de la fête de la Translation ( 11 juillet). C'est après l'office nocturne, alors qu'il est resté à prier auprès des reliques, que ce moine qui s'était endormi vit en songe « un vieillard d'allure distinguée et d'aspect vénérable » qui lui dit :

Ne t'étonne pas de me voir ici, et sois tout à fait certain que je m'appelle frère Benoît et que je suis le patron et le protecteur de ce lieu. Transmets mes salutations aux frères rassemblés, et qu'ils sachent que je serai présent à la célébration de la messe solennelle, mais je n'ai pas pu assister à la psalmodie de l'office nocturne. En effet, j'ai obtenu du Seigneur, pour le frère Aymeri qui était en train de mourir en [Grande]Bretagne, le pardon de ses péchés, je l'ai arraché aux emprises de l'ennemi et j'ai réussi à l'élever jusque dans les demeures de la vie éternelle. Qu'ils sachent donc, par ton intermédiaire, que depuis que, par une disposition divine, ma présence corporelle a été transférée des régions de l'orient jusqu'à ces contrées occidentales, aucun d'entre eux ne subira la damnation, mais que le Christ, les a gratifiés de la récompense de la vie éternelle et les réunira dans les lieux sublimes de la patrie céleste.

Effectivement saint Benoît manifestera sa présence au cours de la messe, et le récit continue :

pour manifester l'étendue de son pouvoir et pour la joie de tous, il manifesta sa présence par la puissance de l'action divine. En effet, alors que tous s'attendaient à voir ou à apprendre quelque chose d'important, il arriva, après la lecture du saint évangile, que tous ceux qui souffraient de quelques maux et se trouvaient à l'intérieur de la sainte basilique purent rentrer chez eux avec le don d'une santé retrouvée. Soixante personnes, en effet, de l'un et l'autre sexe et de tout âge, furent guéries et rendirent en commun et avec éclat des louanges et des actions de grâce à Dieu et au bienheureux père Benoît. À la suite d'une si grande et parfaite action de Dieu, il fut évident pour tout le monde que notre Benoît était présent à la célébration de la messe4.

6 Que s'est-il passé exactement ? il nous est difficile de le cerner, mais il a bien dû se passer quelque chose d'extraordinaire car cet événement a tellement impressionné les esprits qu'il sera repris un siècle plus tard par le moine Jean de Salerne, biographe d'Odon de Cluny, à la gloire de son héros. Cette fois c'est à la suite de la réforme opérée par Odon, vers 930, que saint Benoît, qui avait quitté Fleury pour manifester sa réprobation de la conduite irrégulière des moines, fait sa rentrée solennelle au cours de la grand messe de la fête de la Translation, après avoir promis dans une vision, comme dans l'épisode des Miracula, que tous les frères qui mourront dans ce monastère seront reçus dans le repos éternel ${ }^{5}$.

7 À travers ses reliques, c'est saint Benoît lui-même qui est présent à Fleury, et cette présence est interprêtée de façon si concrète que, dans la même Vie d'odon, Jean de 
Salerne, après avoir raconté une vision par laquelle saint Benoît déclare quitter ce monastère où les moines, par suite de leur mauvaise conduite, ne le laissent pas reposer en paix, nous décrit les moines de Fleury qui, au lieu de se livrer à la prière avec larmes, montent à cheval et se lancent à la poursuite du saint sur tous les chemins des environs ${ }^{6}$ !

Lorsque, à l'occasion d'une épidémie du mal des ardents dans la région de Limoges, des reliques de saint Benoît sont transportées depuis le monastère de Saint-Benoît du Sault, prieuré dépendant de Fleury, André nous dit que : «la rumeur court que notre père Benoît traverse ces régions » et il nous rapporte la prière d'une femme paralysée : « Hélas, très saint Benoît, quelle joie ce serait pour une malheureuse comme moi si elle pouvait se joindre à ton chemin !». Et la pauvre femme fut guérie sur le champ ${ }^{7}$.

Saint Benoît est donc réellement présent dans son monastère et, à plusieurs reprises dans les Miracula nous voyons l'un ou l'autre membre de sa famille s'adresser à lui avec grande familiarité comme à un vivant. Voici, par exemple, l'histoire du moine Christian, sacristain de la basilique de Fleury, qu'Adrevald présente comme un homme simple et rustique. Christian avait fait l'acquisition «d'anneaux de bronze ciselé et doré, attachés aux cordes avec lesquelles, selon la coutume, on sonnait les cloches aux heures fixées pour la célébration de l'office ». Or, une nuit, des voleurs dérobèrent ces anneaux. Le matin venu, Christian découvrit le larcin, et, continue Adrevald :

Comme il croyait fermement que saint Benoît l'écoutait, s'exprimant en langue vulgaire à cause de sa rusticité, car il était germain de naissance, il commença à dire avec grande simplicité : «O saint Benoît, pourquoi dors-tu ainsi avec paresse? Comment veilles-tu avec tant de négligence sur ta maison que de permettre à des voleurs d'emporter furtivement ce que je t'ai apporté pour t'honorer? Certainement cela m'importerait peu qu'il t'enlèvent même tes chausses, toi qui n'as pas défendu ces anneaux. Crois-moi, si tu ne me restitues pas tes anneaux, je ne t'allumerai pas même un cierge ». Après l'avoir menacé ainsi, et par bien d'autres paroles, et avoir même frappé avec un bâton la pierre placée devant le tombeau, il partit tout triste.

Mais les voleurs eurent l'imprudence de revenir une autre nuit et cette fois furent pris par les familiers au service du sacristain. Celui-ci, après leur avoir adressé une sévère réprimande et leur avoir fait remarquer que, selon le droit, ils méritaient la pendaison, " ordonna de les réconforter en leur donnant à manger et à boire et de les renvoyer libres chez eux, leur interdisant de jamais plus oser entreprendre de telles actions ${ }^{8}$. "

Dans le même registre, on peut encore citer la réprimande d'un jeune chevalier qui avait confié son cheval et ses armes à saint Benoît avant de rentrer prier dans l'église d'un monastère consacré à ce saint et ne les retrouve pas en sortant « Eh bien, s'écrie-til, ô très saint Benoît, comment te charges-tu de présenter au Christ les prières de ceux qui t'implorent, que tu n'es même pas capable de te faire le gardien d'un quadrupède? Je te certifie que je ne partirai pas d'ici que tu ne m'aies restitué ce que j'ai perdu par suite de ta négligence. » Inutile de dire que le cheval ne tarda pas à revenir de luimême ${ }^{9}$. Ou encore, dans le même chapitre l'invective d'un clerc qui, entré dans la même église pour prier reçoit sur le front et les yeux la déjection d'un oiseau : «Saint, Benoît, saint Benoît, s'écrie-t-il je te jure que je ne franchirai jamais plus, tant que je vivrai, les portes de ta basilique, si tu ne te manifestes pas comme juge de cette injure ». Et aussitôt l'oiseau tomba mort ${ }^{10}$.

11 Le monastère de Fleury est la propriété personnelle de saint Benoît et tous les biens qui en dépendent sont les biens de saint Benoît. Ainsi lorsqu'une bande de soldats pillards 
s'apprête à s'emparer d'un troupeau de bovins "destiné à servir aux nécessités quotidiennes des frères ", le paysan qui en avait la garde leur dit "qu'il ne fallait pas toucher au troupeau de saint Benoît et que, s'ils le faisaient, ils ne resteraient pas impunis. » Évidemment les pillards ne tinrent aucun compte de l'avertissement et l'un d'entre eux, tirant son épée, essaya de frapper une vache particulièrement grasse, mais son bras resta figé et il fallut que ses compagnons le conduisent devant les reliques de saint Benoît pour qu'il en retrouve l'usage. Et, conclut Adrevald : «il retourne vers les siens et proclame partout ouvertement qu'il y a en ce lieu une vertu divine particulière et que personne ne restera sans punition s'il a molesté saint Benoît en quelque chose $^{11}$.»

Tout au long des divers livres des Miracula, on nous parle des possessions de l'abbaye de Fleury comme de possessions personnelles de saint Benoît. On parle ainsi de la forêt de saint Benoît ${ }^{12}$, de l'avoine de saint Benoît qu'un chevalier téméraire voulait prendre pour nourrir son cheval ${ }^{13}$, de la terre de saint Benoît à Saint-Benoît-du Sault ${ }^{14}$, du petit domaine (praedolium) donné au père Benoît à Ausson (Yonne) ${ }^{15}$, d'un pré de saint Benoît qu'un chevalier veut faire faucher pour ses chevaux ${ }^{16}$, de Bouzonville (Loiret) " possession de notre saint père ${ }^{17}$. À deux reprises il est question de dons faits par des seigneurs : c'est "à saint Benoît et aux frères du monastère de Fleury " que le comte d'Autun Eccard abandonne son domaine de Perrecy-les-Forges ${ }^{18}$, et c'est "au Législateur » qu'un chevalier, nommé Hildebert, abandonne des biens situés aux confins de l'Aquitaine ${ }^{19}$.

13 Les hommes qui vivent sur les terres de saint Benoît, les serfs en particulier, sont membres de la familia sancti Benedicti et celui-ci les prend sous sa protection. Ainsi cet homme du village de Poilly-lez-Gien « de la famille de notre Père » dont la maison faite d'osier et de genêts est la proie des flammes, mais « Dieu, incliné à la miséricorde par les mérites du très saint père Benoît, éteignit le foyer et la cabane fut sauvée, seule une paroi d'osier fut brûlée, et tout le reste demeura intact $\aleph^{20}$. Ou encore l'épisode de l'enfant emporté par un loup au village de La Cour-Marigny ${ }^{21}$, la malheureuse mère invoque saint Benoît "pour qu'il vienne à son secours et lui rende son fils en ne permettant pas que son serviteur soit emporté hors des limites de son territoire. Et, par la clémence de la divine majesté et l'intervention du bienheureux confesseur, ce qu'elle demandait lui fut accordé. Le loup, en effet, arrivant aux limites du territoire de saint Benoît, lâcha le petit enfant sain et sauf et, se dirigeant vers la forêt, regagna sa tanière $\aleph^{22}$. Pourtant il ne fait pas bon à un serf de la famille de saint Benoît de vouloir s'émanciper comme le prouve l'aventure arrivée à «un homme appelé Aubry, de condition servile et de la famille du père Benoît » qui refusait de payer le cens de la servitude. Au cours d'une vision nocturne, son pouce est replié sur ses autres doigts et demeure ainsi pendant quatre mois jusqu'à ce que, par l'intervention de saint Benoît, il soit miraculeusement guéri, gardant toutefois une cicatrice « témoignage perpétuel en avertissement au rebelle $»^{23}$. Mais on peut aussi appartenir à la famille de saint Benoît sans être de condition servile, comme le prouve l'aventure d'Étienne, unus de familia, equestris ordinis vir, qui ayant perdu la raison, fut guéri devant l'autel de la Mère de Dieu, après que ses amis eurent imploré «l'incommensurable clémence du père Benoît ${ }^{24}$.

Serfs ou serves qui appartiennent à la famille de saint Benoît en sont fiers et ne manquent pas de célébrer au cours de l'année les trois fêtes de leur père : le Transitus (21 mars), la Translation (11 juillet) et l'Illation (4 décembre) qui commémore le retour 
des reliques après les invasions normandes. Or un 11 juillet, alors que les habitants de Bouzonville ${ }^{25}$, localité « qui était possession de notre saint père Benoît », célébraient la fête, une jeune fille "qui n'était pas originaire de la famille du saint père » en prit prétexte pour continuer à travailler et saisissant son colum cum fuso elle parcourait en travaillant les rues du village malgré les objurgations des autres femmes qui lui reprochaient de violer une si grande fête. Mal lui en prit car son fuseau lui échappa des mains et la frappant violemment à la mâchoire lui brisa les dents. Mais, sur la suggestion des autres femmes, elle promit de se livrer comme serve du saint père Benoît et d'observer désormais ses fêtes, et aussitôt « elle fut libérée tant de sa douleur aux dents que de sa peur ${ }^{26}$.

La guérison des diverses maladies physiques, en particulier des infirmités devant lesquelles la médecine du temps se montrait totalement impuissante, telle la paralysie ou la cécité, est l'un des principaux bienfaits que l'on attendait des saints patrons et saint Benoît ne manque pas d'exaucer ceux qui se confient à son intercession. Dès son arrivée en Orléanais, avant même d'atteindre Fleury, saint Benoit manifeste son patronage en guérissant un aveugle et un paralytique ${ }^{27}$. Tout au long des neuf livres des Miracula les auteurs nous rapportent des guérisons de paralytiques, on en compte une vingtaine, pour cinq à six guérisons d'aveugles ou de muets. Ces guérisons de paralytiques sont toutes décrites à peu près selon le même schéma: L'infirme, incapable d'utiliser ses jambes, se traîne péniblement sur le sol, ou bien est porté par ses proches devant les reliques du saint. Il reste là en prière pendant parfois plusieurs jours. Puis la guérison est décrite, presque toujours dans les mêmes termes. Voici, par exemple, ce qui nous est dit d'un enfant infirme :

Il resta là tout le jour, et le lendemain, en notre présence et en celle de tous les frères, car c'était l'heure où se célébrait l'office du soir, ses nerfs, longtemps contractés, commencèrent à se détendre, et le canal desséché de ses veines à s'humecter par le passage du sang, enfin ses chevilles, solidement attachées à ses fesses, commencèrent à s'en détacher. En peu de temps l'enfant pût s'étendre et se coucher de tout son long sur le sol. Et peu après, il se releva guéri et se mit à courir librement en tous sens, bien que d'un pas encore titubant ${ }^{28}$.

Les moines de Fleury étaient sincèrement persuadés que saint Benoît avait lui-même voulu le transfert de ses restes depuis le Mont-Cassin jusqu'aux rives de la Loire. Il suffit pour s'en convaincre de relire le discours qu'Adrevald prête à l'abbé Médon lorsque l'évêque de Rouen, Rémi, frère naturel de Pépin-le-Bref, se présenta au monastère avec la mission de prendre les reliques de saint Benoît pour les restituer au Mont-Cassin, conformément à la demande expresse du pape Zacharie :

Le très saint père a lui-même permis, de son propre mouvement, qu'on le transporte ici, car c'est lui qui, par une sainte vision, a invité ses serviteurs à effectuer sa translation. Nous avons reçu, indignes que nous sommes, le saint corps de cet excellent père, nous avons reçu ses précieux restes et jusqu'à présent nous avons veillé à les conserver avec dévotion. Mais s'il lui plaisait, par suite de nos péchés, de quitter les Gaules et de regagner son pays natal, je le déclare clairement, c'est à lui de le manifester et je ne veux pas que nous y fassions obstacle d'aucune façon. Par contre si, comme nous le croyons au plus profond de notre cœur, c'est par la volonté de Dieu et de la sainte providence qu'il a quitté sa patrie et volontairement gagné, pour le salut de nombreuses âmes, ces contrées éloignées situées près des rivages de l'océan, soyez tout à fait certains que vous retournerez chez vous sans avoir pu réaliser votre dessein ${ }^{29}$. 
Et de fait, saint Benoît manifesta clairement sa volonté de demeurer à Fleury car, à peine l'évêque Rémi a-t-il pénétré dans la basilique Notre-Dame où sont conservées les reliques de saint Benoît :

Ils furent frappés d'une si soudaine cécité qu'ils ne pouvaient ni distinguer mutuellement leurs visages, ni savoir où se diriger, sinon à tâtons. Enveloppés de terreur, ils n'attendaient plus rien d'autre que la mort: Les uns se prosternent à terre pour implorer la miséricorde de Dieu, les autres, cherchant le salut dans la fuite, courrent de ci de là et poussent des cris en appellant au secours. Alerté par le tumulte qu'ils faisaient, l'abbé accourt avec les frères et, leur donnant la main, il tire hors du saint temple les profanateurs de l'église qui ont suffisamment et même abondamment reçu leur punition. Prosternés à terre, ils lui demandèrent pardon, il le leur accorda avec indulgence et leur concéda même avec bienveillance des reliques du corps de ce très glorieux confesseur du Christ. Après les avoir restaurés et les avoir somptueusement munis de tout le nécessaire pour le voyage, il les renvoya chez eux ${ }^{30}$.

17 Au milieu du XI ${ }^{\mathrm{e}}$ siècle, l'auteur anonyme de la finale du livre VII des Miracula reviendra sur ce sujet. Se basant sur le témoignage de l'abbé de Saint-Riquier, Gervin ${ }^{31}$, qui en avait fait le récit devant l'abbé de Fleury Rainier ${ }^{32}$, l'abbé de Saint-Denis Hugues ${ }^{33}$ et l'ancien abbé de Saint-Médard Rainaud, l'auteur nous raconte que les moines du MontCassin, attristés par la perte des reliques de leur père, avaient décidé de faire un jeûne de trois jours pour prier Dieu de leur manifester sa volonté en la matière. Or, le troisième jour, saint Benoît lui-même apparut à l'abbé du Cassin, Richard ${ }^{34}$ et lui dit :

Je suis le frère Benoît, que Dieu a donné à ce lieu comme intendant et défenseur [...] Tu désirerais, si cela était permis, posséder mon corps, mais il n'est pas permis de violer le temple de mon sépulcre ; c'est en effet par l'action et la volonté de mon Dieu tout puissant qui ordonne toutes choses selon sa prescience, et par le propre choix de ma volonté que mes ossements reposent, dignement inhumés, au lieu de Fleury, dans les Gaules. Par ailleurs, sachez que je suis toujours personnellement présent chez vous car la très clémente compassion du Dieu très miséricordieux a confié à ma protection la garde de ces deux lieux. Aussi, puisque l'un et l'autre lieu sont confiés à ma garde, j'en prends soin de jour et de nuit par une constante prévoyance et je me tiens toujours devant Dieu en intercesseur infatigable pour ces deux lieux qui me sont confiés ; sur les deux, en effet, demeure une seule gloire, une seule prévoyance, une seule protection, une seule défense. Conduisez-vous donc virilement, vous et eux, et que votre cœur se conforte dans le Christ, gardant indéfectiblement les préceptes de la sainte règle, afin que, par l'intercession de mes prières, vous méritiez de demeurer toujours heureux dans la félicité du Christ. Qu'il vous suffise de me savoir toujours spirituellement présent, et prenez garde à ne jamais vouloir violer mon sépulcre ${ }^{35}$.

18 Le premier devoir d'un patronus à l'égard de sa familia est de protéger tous les membres de la famille ainsi que leurs biens, et tout au long des Miracula, on montre saint Benoît s'acquittant de cet office de protecteur. Ainsi, lorsque, un jour de fête de l'Illation, on manque de poisson pour restaurer dignement les nombreux pèlerins accourus, c'est saint Benoît qui intervient, de sa propre initiative, pour tirer les moines d'embarras :

Peu avant les vigiles de la dite fête, un frère qui, selon la coutume, reposait sur son lit pendant la nuit, se vit transporté en songe sur la Loire, au lieu où la rivière qu'on appelle couramment Obla se jette dans la Loire. Lui-même se tenait sur la rive de cette rivière, et, sur l'autre rive du fleuve, se tenait un clerc d'aspect vénérable qu'il ne connaissait pas. Ce clerc tenait en main un bâton incurvé, semblable à la crosse des anciens évêques, à l'aide duquel il faisait sortir des profondeurs de l'eau un grand troupeau de porcs qu'il poussait avec grand bruit vers le lieu où semblait être le moine. Une fois réveillé, dès la fin de l'office nocturne, ce moine appelle discrètement le doyen du monastère, il lui raconte son songe et lui conseille d'aller 
avec des frères pêcher avec confiance dans le fleuve. Il est certain, dit-il que le bienaimé soldat du Christ, va accorder à ses frères une très abondante pêche de poissons. C'est certainement cela que signifiait son songe, les porcs représentaient les poissons, et le personnage inconnu mais d'aspect vénérable, le grand saint Benoît. Réconfortés par cette vision, les frères préparent à l'envi les filets et courrent vers le fleuve: on jette les filets et on tire de l'eau un énorme ban de poissons. La pêche fut même si abondante qu'on fut obligé d'aller chercher un chariot pour la transporter. Et l'on reconnut ainsi que le bienheureux père était capable de venir en aide à ses disciples tant qu'ils restaient inébranlablement fidèles à tous les commandements du Seigneur, lui qui, portant encore le fardeau de l'humaine faiblesse, avait pu secourir ses disciples manquant de pain par un cadeau de deux cents deniers de farine ${ }^{36}$.

19 Un autre jour, saint Benoît intervient pour libérer un bateau chargé de sel qui remontait la Loire depuis Nantes et que le comte d'Orléans avait fait arrêter et prétendait soumettre à l'impôt du tonlieu malgré l'exemption de tout droit accordée par privilège royal.

Cela se passait un dimanche. Mais, vers la troisième heure du jour, alors que tous assistaient à la célébration de la messe, le bateau, qui était consigné avec les autres au port de la douane, s'échappa soudain du port sans l'aide d'aucun rameur, gagna le milieu de la Loire, à l'endroit où le courant est le plus fort, et là, naviguant vigoureusement contre le courant contraire, il parvint à la poterne, dite encore aujourd'hui, poterne de Saint Benoît, et y aborda. De toute la ville, on accourt voir ce prodige inouï et, avec stupéfaction, on pousse des cris et on s'interpelle, disant : «Venez, amis, regardez comment ce nouveau marinier, Benoit aimé de Dieu, a piloté son bateau au milieu des flots sans l'aide d'aucun rameur!» Toute l'audace des fonctionnaires du fisc en fut confondue, et cela mit fin à leur folie ${ }^{37}$.

Saint Benoît intervient aussi en faveur des membres de sa famille, des plus démunis en particulier, lorsque ceux-ci sont dans le besoin. On a déjà vu son intervention pour délivrer un enfant enlevé par un loup. Voici maintenant comment, par pitié pour une pauvre veuve indigente, saint Benoît intervint pour sauver de la pendaison le fils de cette femme qui avait pris l'habitude de voler pour pouvoir porter secours à sa mère :

Une vieille femme, de la famille de notre père, dépourvue de tout le nécessaire, n'avait d'autre secours dans sa viduité et sa vieillesse que son fils. Mais celui-ci n'avait pas de quoi subvenir au dénûment de sa mère, car il n'arrivait pas à lui procurer les aliments et les vêtements nécessaires par le seul travail de ses mains, aussi décida-t-il d'y suppléer par des larcins. [Mais, un jour, il est pris sur le fait, en compagnie d'un autre voleur, et tous deux sont condamnés à la pendaison.] Cette vieille femme, apprenant que son fils avait été condamné à la pendaison, se précipite à l'église, toute hors d'haleine, et interpelle saint Benoît : « Mon seigneur Benoît, dit-elle, que t'a fait ta misérable servante? alors que je n'ai aucun autre secours, tu le sais bien, que mon fils qui maintenant est conduit au supplice. Tu sais, seigneur, que c'est poussé par la nécessité, qu'il commettait ces vols. Rends-moi, mon seigneur, celui qui est mon fils et ton serviteur et qui vient d'être arrêté, qu'il pourvoie à mes malheurs afin que ta servante ne meure pas de faim ». [...] Le bienheureux père, incliné à la miséricorde par la supplication de cette vieille, réalisa un prodige dont on n'a encore jamais entendu parler. Les deux jeunes gens, conduits au lieu destiné au supplice, sont suspendus à des potences. L'un des deux, le compagnon du fils de la veuve, perdit la vie à l'instant. Mais, ce dernier, suspendu depuis le lever du soleil jusqu'à la troisième heure ne put pas mourir. [...] On comprit alors que c'était grâce aux larmes et aux prières de cette veuve qu'on avait vu se précipiter à l'église tout en larmes, que le Dieu tout puissant avait conservé la vie à son fils par les mérites du père Benoît qu'elle avait invoqué avec dévotion, et on magnifia la clémence du Tout Puissant. Quant au jeune homme, interrogé il 
déclara n'avoir senti aucune lésion pendant tout le temps de sa suspension. Aussi comprit-on que c'était plus par indigence que par malice qu'il avait volés ${ }^{38}$.

21 À deux reprises, on nous montre même saint Benoît intervenant en personne au cours d'un combat pour assurer la victoire à ceux qui se battent pour la protection de ses biens. La première fois, c'est au temps des invasions normandes. Les moines de Fleury, avertis de l'arrivée imminente des pirates normands, ont quitté le monastère, enmenant avec eux jusqu'à leur domaine de La Cour-Marigny, entassé sur des charriots, tout ce qu'ils pouvaient emporter. Mais les Normands, voyant dans le sol les ornières laissées par les lourds charriots, se lancent à leur poursuite. C'est alors qu'intervint Hugues l'Abbé qui passait par là à la tête d'une petite troupe :

Il arrivait près du monastère et apprit par les siens que les frères étaient poursuivis par les ennemis et seraient très bientôt pillés s'ils n'étaient pas délivrés par une intervention merveilleuse de Dieu. Ayant donc appris cela, il pesa le pour et le contre, car il n'avait avec lui qu'un tout petit nombre de soldats et il était inquiet et soucieux, se demandant comment il pourrait s'opposer à une telle multitude. Pourtant, sur les encouragements de Girbold, le très noble comte d'Auxerre, qui affirmait qu'aucun mal ne pourrait arriver à qui interviendrait contre l'armée des ennemis pour venir au secours de saint Benoît, il prit la décision d'engager le combat. Leur courage affermi par cette exhortation, et confiants en l'aide de cet excellent père, ils poursuivirent les ennemis sur leurs arrières, non loin du monastère. Un violent combat s'engagea avec grande ardeur, et ils firent furieusement un si grand carnage des ennemis que, sur une si grande multitude, c'est à peine s'il en resta un pour raconter à la postérité l'issue de la bataille. Après avoir obtenu cette victoire si désirée, le chef de guerre interrogea les siens, leur demandant si quelqu'un avait vu de ses yeux un moine d'aspect vénérable lui ouvrant le chemin au milieu de la multitude des ennemis. Ils lui répondirent n'avoir vu aucun moine dans ce combat. Lui alors leur dit : «Pendant tout ce combat, saint Benoît m'a protégé, tenant de sa main gauche les rênes de mon cheval, il me dirigeait et me protégeait, tandis que de sa main droite il tenait un bâton avec lequel il mit à mort beaucoup d'ennemis en les assommant». Ainsi, grâce à l'intervention de notre père saint Benoît, les méchants reçurent leur châtiment et les innocents retrouvèrent la tranquillité, par la force du béni Fils de Dieu dont le nom demeure béni dans les siècles des siècles ${ }^{39}$.

22 Une autre fois, c'est, sous la plume d'André de Fleury, au cours du XI ${ }^{\mathrm{e}}$ siècle, parmi une série de faits merveilleux qui se situent au prieuré de Châtillon-sur-Loire ou dans ses environs. Au cours d'un combat entre Gilon, seigneur de Sully qui deviendra moine à Fleury sur la fin de sa vie, et Landry, comte de Nevers. Gilon s'élance au combat avec les siens en criant, mot à mot en beuglant (Benedictum ore se boare), le nom de Benoît et il vient facilement à bout d'une troupe beaucoup plus nombreuse que la sienne ${ }^{40}$.

Cette protection que saint Benoît accorde à ceux qui se confient à son patronage, se traduit souvent par une punition infligée à ceux qui ont la témérité de vouloir leur nuire. Nos auteurs insistent même volontiers sur ces punitions et en multiplient les exemples, aptes à inspirer une crainte salutaire à qui serait tenté d'imiter les pillards des biens de saint Benoît.

24 Adrevald nous raconte ainsi une curieuse histoire à propos d'une controverse entre l'avoué de Saint-Benoît et celui de Saint-Denis au sujet de serfs dont on se disputait la propriété :

Comme les juges faisaient traîner le procès trop longtemps, car les uns ne voulaient pas céder aux autres, ni ces autres donner leur accord aux premiers, il fut décidé que de l'une et l'autre partie on choisirait des témoins qui, après avoir prêté serment, mettraient fin à la controverse en combattant avec des boucliers et des 
bâtons. Et cette décision parut juste et correcte à tout le monde. Mais un légiste du Gâtinais qui, par une sorte de présage, avait reçu un nom de bête au lieu d'un nom d'homme, était venu après avoir été corrompu par un présent de l'avoué de Saint Denis. Craignant que si les deux hommes luttaient entre eux, leur témoin ne soit battu, il émit l'avis qu'il n'était pas convenable que des témoins décident par les armes dans des affaires ecclésiastiques, mais qu'il fallait que les avoués partagent entre eux. Le vicomte Génésius, se rangeant à cet avis, dit qu'il était plus équitable de partager les serfs, plutôt que de s'en remettre au combat des témoins, et toute l'assemblée se rangea à son avis. Mais saint Benoît n'oublia pas ce juge et légiste qui le premier, avec ruse et de façon bestiale conformément à son nom, avait donné l'avis de partager les serfs. Car, aussitôt que ces serfs eurent été divisés en deux parts, cet homme fut frappé par un juste jugement de Dieu, et perdit complètement l'usage de la parole, de sorte qu'il ne pouvait plus rien dire. Ses familiers qui l'accompagnaient, comprenant le sens de cet événement, le conduisirent jusqu'au monastère du saint confesseur du Christ qu'il avait si gravement offensé. Il y resta près d'un mois, demandant par signes, autant qu'il en était capable, l'aide du vénérable père et il reçut enfin un retour partiel à la santé ; mais tant qu'il vécut, il ne lui fut plus jamais possible de prononcer le nom de saint Benoît ${ }^{41}$.

Ici le coupable, après avoir reçu la punition méritée, est guéri, partiellement du moins, mais le plus souvent la punition encourue est tout simplement la mort. Voici au moins un de ces épisodes, l'un des mieux connus de nos jours encore, puisque la tête du coupable est, pour mémoire éternelle, gravée dans une pierre de la basilique de SaintBenoît-sur-Loire. À l'annonce de l'arrivée d'une troupe de Normands, les moines s'étaient, cette fois encore, enfuis, emportant avec eux tout ce qu'ils avaient pu entasser sur des charriots. Le chef de la troupe qu'Aimoin qualifie de roi, un certain Rainaldus, qui s'était installé dans le dortoir des frères, eut, pendant son sommeil, une vision de saint Benoît qui l'interpella en ces termes :

«En quoi t'ai-je offensé, Rainaldus, pour que tu viennes me troubler, moi et les miens, dans notre propre demeure? Mais sache que désormais je prendrai soin et de t'arrêter dans tes projets et de rendre aux serviteurs du Christ, ainsi qu'à mes ossements, la paix dont ils ont besoin ». Ceci dit, il toucha la tête du roi encore endormi avec le bâton recourbé qu'il tenait en main, lui annonça la fin prochaine de sa vie et se retira.

Sans plus tarder, Rainaldus ordonna de décamper et de regagner le sol natal, mais à peine arrivé chez lui, il perdit la vie au cours d'une terrible tempête ${ }^{42}$.

Si saint Benoît est le patron et est personnellement propriétaire de tout ce que possède l'abbaye, il peut aussi déléguer l'un ou l'autre des saints dont le monastère possède également les reliques, comme en témoigne un curieux épisode du conflit qui opposa aux abbés de Fleury, Oilbold et Abbon, l'évêque d'Orléans Arnoul, dont Aimoin nous dit qu'il était: "par ailleurs vraiment bon, bien instruit des lois ecclésiastiques et les observant fidèlement, mais qui n'a jamais vraiment aimé les prélats de ce lieu de Fleury ». Le conflit portait, cette fois, sur une vigne située tout près d'Orléans et qui avait été occupée indûment par des serviteurs de l'évêque. Celui-ci n'ayant accordé aucune attention aux réclamations de l'abbé Oilbold, ce dernier, après avoir pris conseil des anciens, décida d'envoyer quelques frères faire la vendange sous la protection des reliques des saints. On ne touche pourtant pas aux reliques de saint Benoît qui demeurent à Fleury, mais on emporte, sub nomine inclyti confessoris Dei Benedicti, les reliques des saints martyrs Maur et Frogent ${ }^{43}$.

Et, pendant le trajet, à ceux qui, voisins ou habitant le long du chemin, demandaient de qui était ce si solennel cortège qui passait, on répondait, de saint Benoît. Parmi ceux-ci, un malade, affaibli par les brûlures des fièvres, demanda à ceux qui 
passaient en l'honneur de qui une telle foule encombrait le chemin derrière les croix. On lui répondit que saint Benoît, bien-aimé de Dieu, passait par ce chemin se rendant dans les faubourgs d'Orléans pour prendre la défense de ses vignes.

Ainsi protégés par saint Benoît, ou par les reliques des saints qu'il avait délégués, les moines vendangèrent en paix tandis que le malade était guéri ${ }^{44}$. Et on peut faire la même remarque au sujet des prodiges qu'André raconte dans le livre V des Miracula, prodiges qui se passent à Châtillon-sur-Loire ou dans les environs par l'intermédiaire des reliques de saint Posen ${ }^{45}$.

Dans tout ceci, le théologien ne peut relever aucune trace de superstition, de religion populaire ou d'attribution aux saints de pouvoirs autrefois reconnus aux dieux païens. Saint Benoît est toujours présenté dans le rôle d'intercesseur et c'est Dieu, on précise parfois le Christ, qui accomplit le prodige. Dès la première ligne de son prologue aux Miracula, Adrevald enlève toute ambiguité : « Ci-commencent les miracles de l'excellent confesseur du Christ Benoît, miracles que la puissance divine opéra par lui après son trépas ${ }^{46}$. " Dans l'impossibilité de tout citer, notons entre autres cette réflexion attribuée aux envoyés que Pépin-le-Bref avait chargés de récupérer les reliques de saint Benoît : «Et eux, de retour au palais, racontèrent au très excellent roi Pépin la grande puissance de Dieu à l'œuvre en ce lieu par l'intercession et les mérites de la sainte mère de Dieu et toujours vierge Marie, et de saint Pierre, prince des apôtres, et enfin du glorieux père Benoît. » Enfin André de Fleury, terminant le troisième livre des Miracula, parle des «miracles que Dieu a daigné opérer en divers lieux par les mérites de saint Benoît. "

C'est bien là le rôle que le grand docteur d'Occident, Augustin, attribuait aux saints : «Les prières des fidèles, écrit-il, demandent aux saints d'assumer les défunts et de les assister devant le Seigneur en tant que patrons ${ }^{47}$. Commentant ce texte, Yvette Duval écrit : «Ce patrocinium est le fondement du culte des saints, protecteurs et intercesseurs auprès de Dieu de tous les fidèles, morts et vivants, pour lesquels on les invoque et les prie $^{48}$. »

Les neuf livres des Miracula sancti Benedicti ont été écrits pour rappeler à la postérité combien est efficace la puissance d'intercession de saint Benoît auprès du Dieu toutpuissant.

\section{NOTES}

1. P. Brown, Le culte des saints, son essor et sa fonction dans la chrétienté latine, Traduit par A. Rousselle, Paris 1984, p. 58.

2. B. Beaujard et F. Prévot, «Le culte des saints en Occident ", Histoire du christianisme, 3, sous la direction de J.-M. Mayeur, Ch. et L. Pietri, A. Vauchez \& M. Venard, Paris, 1998, p. 1062.

3. Une édition critique des Miracula sancti Benedicti, avec traduction française, est actuellement en préparation par mes soins en collaboration avec Mme Cécile Dejardin-Bazaille, à paraître dans la collection Sources d'Histoire Médiévale publiée par l'Institut de Recherche et d'Histoire des Textes. Dans le cours de cet article, les références seront données à la précédente édition : Les 
miracles de saint Benoît écrits par Adrevald, Aimoin, André, Raoul Tortaire et Hugues de Sainte Marie, moines de Fleury, réunis et publiés par E. De Certain, Paris, 1858.

4. I, 40, De Certain, pp. 84-85.

5. PL 133, col. 82C-83C.

6. PL 133, col. 80C-D.

7. IV, 1, De Certain, pp. 176-177.

8. I, 26, De Certain, pp. 58-60.

9. IV, 8, De Certain, pp. 183-184.

10. IV , 8 , De Certain, pp. 184-185.

11. I, 27, De Certain, pp. 62-63.

12. II, 8, De Certain, p. 109.

13. II, 14, De Certain, p. 116.

14. II, 15, De Certain, p. 118.

15. VIII, 17, De Certain, p. 300.

16. VIII, 18, De Certain, p. 302.

17. VIII, 32, De Certain, p. 329.

18. III, 15, De Certain, p. 161.

19. IV, 5, De Certain, pp. 179-180.

20. I, 37, De Certain, pp 79-80.

21. La Cour-Marigny, canton de Lorris, Loiret, en lisière de la forêt d'Orléans.

22. I, 39, De Certain, pp. 82-83.

23. V, 8, De Certain, pp. 205-207.

24. VIII, 31, De Certain, p. 328.

25. Bouzonville-aux-Bois, Loiret, canton de Pithiviers.

26. VIII, 32, De Certain, pp. 329-330.

27. HT 9,10, De Certain, p. 9.

28. I, 29, De Certain, p. 66.

29. I, 16, De Certain, p. 40.

30. I, 17, De Certain, pp. 41-42.

31. Gervin I ${ }^{\mathrm{er}}$, abbé de Saint-Riquier de 1045 à 1075, présent au couronnement de Philippe I ${ }^{\mathrm{er}}$ à Reims en 1059. Cf. Gallia Christiana Xa, col. 1249-1251.

32. Rainier, abbé de Fleury, présent également au couronnement de Philippe ${ }^{\mathrm{er}}$.

33. Hugues, abbé de Saint-Denis, attesté en 1049, présent également au couronnement de Philippe $\mathrm{I}^{\mathrm{er}}$, décédé en 1061 ou 1062. Cf. Gallia Christiana, VII, col. 363-364.

34. Ricardus, en italien Richerio, moine de Niederaltaich, abbé de Leno, puis du Mont-Cassin de 1038 à 1055. Cf. W. Wuhr, « Die Wiedergeburt Montecassinos unter ersten Reformabt Richer von Niederaltaich », Studi Gregoriani, III, Roma, 1948, pp. 369-450.

35. VII, 15, De Certain, pp. 273-274.

36. I, 22, De Certain, pp. 52-53. Cf. Gregorii Magni Dial. II, 21, 2, SC 260, p. 198.

37. I, 19, De Certain, pp. 46-47.

38. VIII, 44, De Certain, pp. 349-351.

39. I, 41, De Certain, pp. 86-89.

40. V, 15, De Certain, pp. 212-213.

41. I, 25, De Certain, pp. 56-57.

42. II, 2, De Certain, pp. 97-98.

43. Maur, martyr, inconnu par ailleurs, dont les reliques avaient été apportées des catacombes romaines - à ne pas confondre avec saint Maur, disciple de saint Benoît - fête le 22 novembre (BHL 5789-5790). Frogent ou Chrodegant, évêque de Sées, dont les reliques furent transportées à Fleury en même temps que celles de saint Maur martyr, à la fin du $\mathrm{X}^{\mathrm{e}}$ siècle, fête le 3 septembre (BHL 1782-1784). 
44. II, 19, De Certain, pp. 123-125.

45. Saint Posen, personnage mal identifié, patron secondaire du prieuré de Châtillon-sur-Loire (Loiret, chef-lieu de canton, arrondissement de Montargis). Cf. AASS, 3 jun, 412. L'invention de ses reliques est raconté en Mir V, 6, De Certain, p. 203.

46. I, Pr. (omis par De Certain).

47. De cura gerenda pro mortuis, 6, Bibliothèque Augustinienne, 2, II, 1948, p. 474.

48. Y. Duval, Auprès des saints corps et âme, Paris, 1988, p. 183.

\section{AUTEUR}

\section{ANSELME DAVRIL}

OSB, Abbaye de Fleury, Saint-Benoît-sur-Loire 